\title{
Low caloric intake predicted mortality for geriatric patients in hospital
}

\author{
Incalzi RA, Capparella $O$, Gemma A, et al. Inadequate caloric intake: a risk factor for mortality of geriatric patients in the \\ acute-care hospital. Age Ageing 1998 May;27:303-10.
}

\section{Question}

Do geriatric patients in hospital ingest adequate calories and does inadequate caloric intake increase the risk of death?

\section{Design}

Cohort study.

\section{Setting}

Acute care university hospital in Rome, Italy.

\section{Patients}

370 patients $\geqslant 70$ years of age (mean age 79 y, $56 \%$ women) who were admitted to the surgical ward for elective abdominal surgery $(n=86)$; or admitted for acute medical problems to the geriatric ward $(n=135)$ or internal medicine ward $(n=149)$. Patients who gave incomplete dietary information, had terminal illness, or had morbid obesity were excluded.

\section{Assessment of risk factors}

Body mass index (BMI), per cent ideal weight, mid arm circumference, serum albumin, serum lymphocytes, and serum haemoglobin (on admission and discharge); and age, preadmission dependency in Activities of Daily Living (ADL), depression (Geriatric Depression Scale), cognitive status (Mini Mental Status Examination), and comorbidity (Charlson index) (on admission).

\section{Main outcome measures}

Adequate caloric intake was based on an energy requirement of 1.65 times the basal metabolic rate; adequate protein intake was based on a ratio of 627 non-protein $\mathrm{kJ}(150 \mathrm{kcal})$ to $1 \mathrm{~g}$ of nitrogen; and both were adjusted for patients who had a BMI $<22$ or $>30 \mathrm{~kg} / \mathrm{cm}^{2}$, nutrition related disease, or sepsis. Nutritional depletion at discharge was defined as a mid arm circumference decrease of $\geqslant 3.6 \%$ from baseline. Deaths were also recorded.

\section{Main results}

Patients who were admitted to the surgical ward had lower comorbidity and greater use of total parenteral nutrition than those who were admitted to the geriatric or internal medicine wards. The 3 clinical setting groups did not differ in the ratio of actual to adequate caloric intake (range $63 \%$ to $69 \%$ ), ratio of non-protein calories to nitrogen (range 532 to $556 \mathrm{~kJ} / \mathrm{g}$ [127 to $133 \mathrm{kcal} / \mathrm{g}]$ ), or difference in mid arm circumference between baseline and discharge (range -6.9 to $-5.1 \mathrm{~mm}$ ). However, nutritional depletion at discharge was significant for all 3 groups. Lower caloric intake was related to higher age, greater cognitive impairment, greater preadmission dependency in ADL, lower BMI, hypoalbuminaemia, and comorbidity; and mortality was related to depression, preadmission dependency in ADL, hypoalbuminaemia, lymphocytopenia, lower caloric intake, and comorbidity. Caloric intake $<40 \%$ of adequate intake was predicted by preadmission dependency in ADL and low BMI; and mortality was predicted by preadmission dependency in ADL, low caloric intake, lymphocytopenia, and hypoalbuminaemia.

\section{Conclusions}

Nutrition depletion and inadequate caloric intake existed among geriatric patients in hospital. Hospital ward did not predict caloric intake, but preadmission dependency in activities of daily living and low body mass index did. Mortality was predicted by low caloric intake, preadmission dependency in activities of daily living, lymphocytopenia, and hypoalbuminaemia.

\section{Source of funding: no external funding.}

For correspondence: $\operatorname{Dr} R$ A Incalzi, Istituto di Medicina e Geriatria dell'Universita' Cattolica, Policlinico A Gemelli, Largo A Gemelli 8, 00168 Roma,Italy. Fax +3963051911.

\section{Commentary}

This study by Incalzi et al shows the relation between caloric intake and death among geriatric patients in hospital. The findings have implications for nursing practice. The importance of functional status in determining outcomes of care is clearly shown. Patients with functional impairment at the time of hospital admission were more likely to die during their stay in hospital regardless of the reason for admission or the hospital unit to which they were admitted. These patients were also at greater risk for decreased caloric intake, which itself is a risk factor for mortality. The fact that terminally ill patients were excluded from the sample adds weight to the findings. The deaths that occurred may have been avoidable to some extent. The study did not address implications for quality of life. The report may have overestimated starvation rates because the definition of 1.65 times basal metabolic rate may be a high caloric intake for elderly patients on bed rest.

Nurses should assess the functional status of geriatric patients upon hospital admission and identify areas in which nutritional support is needed. This may include providing preferred foods and ensuring nursing staff availability to provide physical assistance with eating. If total parenteral nutrition is used, it should not be stopped prematurely. A referral to a dietitian may be appropriate.

Further research is needed to (a) identify the mechanisms by which impaired functional status leads to increased mortality; (b) identify interventions which diminish the effect of impaired functional status on mortality; (c) identify interventions which diminish the effect of impaired functional status on caloric intake; and (d) determine whether the relation between nutritional status, functional status, and mortality in geriatric patients in hospital is also found in those admitted to nursing homes and assisted living facilities.

Amy Laufer Kenefick, RN, MSN Clinical Assistant Professor School of Nursing University of Massachusetts Amherst, Massachusetts, USA 